\title{
Alterations in the expression of PDCD4 in ductal carcinoma of the breast
}

\author{
YONG HANNAH WEN ${ }^{1}$, XIUQUAN SHI ${ }^{2}$, LUIS CHIRIBOGA ${ }^{1}$, \\ SACHIKO MATSAHASHI ${ }^{3}$, HERMAN YEE ${ }^{1}$ and OLUBUNMI AFONJA ${ }^{2}$
}

${ }^{1}$ Departments of Pathology and ${ }^{2}$ Pediatrics, New York University School of Medicine, 550 First Avenue, New York,
NY 10016, USA; ${ }^{3}$ Department of Internal Medicine, Saga Medical School, Saga University, Saga 849-8501, Japan

Received April 20,2007; Accepted August 16, 2007

\begin{abstract}
Programmed cell death 4 gene (PDCD4), an in vivo repressor of transformation, was originally isolated from a human glioma library by screening it with an antibody against a nuclear antigen in proliferating cells. PDCD4 functions as a transformation repressor by inhibiting the activity of the RNA helicase, eIF4A. We previously showed that retinoids, anti-estrogens and HER2/neu antagonist induce PDCD4 expression in human breast cancer cell lines. Very little is known about the expression of PDCD4 in human breast cancer tissues or the significance of the PDCD4 expression in breast cancer. To gain insight into the pattern of the PDCD4 expression in breast tissues, we performed an immunohistochemical analysis of the PDCD4 expression in 80 archived, normal and ductal breast carcinoma tissues (invasive and carcinoma in situ) (DCIS) and correlated PDCD4 expression with expression of known prognostic markers in breast cancer (ER, PR and HER $2 / \mathrm{neu}$ ). To assess the role of methylation on PDCD4 expression in breast cancer cells, breast cancer cell lines were treated with the demethylating agent 5-deoxy-azacytidine and analyzed for PDCD4 expression. We observed primarily nuclear localization of PDCD4 in ductal carcinoma in situ compared to normal breast tissues where the PDCD4 expression was predominantly cytoplasmic. This was seen more frequently in DCIS cases that were ER positive and HER2/neu negative samples. PDCD4 expression was markedly decreased in the invasive ductal carcinoma. We did not observe any significant relationship between PDCD4 expression and the expression of RAR or PR. In T-47D, MDA-MB-435 and MDA-MB-231 cells, treatment with 5-deoxy-azacytidine did not result in an
\end{abstract}

Correspondence to: Dr Olubunmi Afonja, Department of Pediatrics, New York University School of Medicine, 550 First Avenue, New York, NY 10016, USA

E-mail: afonjo01@med.nyu.edu

Key words: programmed cell death 4, estrogen receptor, HER2/neu, apoptosis, methylation, differentiation increased expression of PDCD4. The present study demonstrated altered cellular localization of PDCD4 when comparing normal breast to neoplastic breast tissues. In addition, there was a decreased expression of PDCD4 in breast cancer when compared with normal breast tissue. A loss of the PDCD4 expression in breast cancer cell lines does not appear to result from hypermethylation of the PDCD4 promoter.

\section{Introduction}

The programmed cell death 4 gene (PDCD4) was originally isolated from a human glioma library by screening it with an antibody against a nuclear antigen in proliferating cells (1). PDCD4 (H731L) is homologous to the mouse Pdcd4 gene (also known as MA-3/TIS/A7-1) (2) and it codes for a protein of 469 amino acids with a predicted size of $62 \mathrm{kDa}(3,4)$. The two human PDCD4 homologs, H731L and H731, are respectively, 96 and $93 \%$ identical to the mouse Pdcd4 gene (5). H731L and H731 are alternative transcripts of the same gene with $\mathrm{H} 731$ lacking 11 amino acids present in the N-terminal region of the mouse Pdcd4 and H731L (5). The human PDCD4 gene was localized to chromosome 10q24 (6) but its function is not well defined. The deduced amino acid sequence of PDCD4 suggests that the protein contains two N-terminal basic domains, which may function as nuclear localization signals and two nuclear export sequences, suggesting that PDCD4 is capable of shuttling back to the cytoplasm under certain conditions (2,7). Pdcd4 is now known to prevent eIF4A from binding to eIF4G, resulting in the inhibition of capdependent translation (5). PDCD4 has an intrinsic RNAbinding activity (7) and it was postulated that it may also be involved in RNA-processing events such as splicing and nucleo-cytoplasmic transport. Expression of the mouse Pdcd4 gene was shown to be associated with apoptosis in several systems $(8,9)$. However, its role in apoptosis is yet to be elucidated.

Very little is known about the upstream regulators and downstream targets of PDCD4 in normal cells. Yang et al, reported that Pdcd4 inhibited the activation of AP-1-dependent transcriptional activity in a dose-dependent manner in colon cancer cell lines (10). The serine/threonine kinase Akt is now reported to be an upstream modulator of the Pdcd4 activity. Akt (protein kinase B) was observed to specifically phos- 
phorylate Ser67 and Ser457 of Pdcd4 in vitro and in vivo resulting in a significant decrease in the ability of Pdcd4 to interfere with the transactivation of an AP-1-responsive promoter by c-Jun (11). Additionally, RKO cells, stably transfected with Pdcd4, had a reduced invasive capability which was associated with a significant reduction in Mitogen-Activated Protein kinase kinase kinase kinase 1 (MAP4K1) activity, thereby suggesting that Pdcd4 targets the Jun N-terminal kinase (JNK) pathway by modulating upstream targets such as MAP4K1 (12). Carbonic anhydrase II was recently identified as a PDCD4 target in endocrine tumor cell lines (13).

PDCD4 is ubiquitously expressed at low levels in normal tissues $(2,8,9,14)$. There are conflicting data about its primary cellular localization and this appears to be dependent on the tissue being analyzed and the cellular microenvironment (7). The PDCD4 expression was detected primarily in nuclear extracts from the T-47D breast cells (15). It was, however, detected within the nucleus and cytoplasm of normal breast epithelial cells $(2,4)$. There is experimental evidence suggesting that PDCD4 is primarily a nuclear protein which is capable of shuttling into the cytoplasmic compartment of cultured cells upon serum withdrawal or stress (7). Together, these data suggest that low levels of functional PDCD4 may be essential for cell proliferation and other physiological processes yet to be identified (4). A loss of the PDCD4 expression or accumulation of protein in the nuclei may positively regulate cell proliferation and this may in part contribute to the tumorigenic process in various malignancies. In our initial studies of the PDCD4 expression in cultured cancer cell lines, PDCD4 expression was not detected in breast cancer cells that lacked ER and RAR expression (15). Our observation of the lack of the basal expression of PDCD4 in most of the ERbreast cancer cell lines suggests that a loss of the PDCD4 expression may contribute to the development of an aggressive phenotype of breast cancer (15).

In this study, we performed an immunohistochemical analysis of the PDCD4 expression in ductal carcinoma of the breast to determine if a loss of the PDCD4 expression correlates with an aggressive histological phenotype. Additionally, we analyzed the effects of a demethylating agent on the PDCD4 expression in cultured breast cancer cells.

\section{Materials and methods}

Breast tissue samples. Formalin-fixed paraffin-embedded ductal carcinomas of the breast and normal breast tissues were retrieved from the archival Surgical Pathology files of the Department of Pathology at Bellevue Hospital/New York University School of Medicine and was approved by the Institutional Review Board. A total of 80 cases were selected, including 65 cases of invasive ductal carcinoma, 5 of which contain associated ductal carcinoma in situ (DCIS) and 10 cases of pure DCIS. Adjacent benign breast epithelium was present in 28 cases. Additionally, 5 cases of normal breast (from reduction mammoplasty) were selected and served as normal controls.

Cell lines. T-47D, MDA-MB-231 and MDA-MB-435 cells were obtained from the American Type Tissue Culture
Collection (ATCC; Rockville, MD). T-47D cells were maintained in RPMI-1640 (Mediatech Inc., USA) supplemented with $10 \%$ fetal calf serum (FCS). MDA-MB-231 and MDA-MB-435 cells were maintained in Leibowitz's L-15 medium supplemented with $10 \%$ FCS.

Immunohistochemistry. Tissue microarrays were constructed with duplicate 3-mm cores taken from different areas of each case to ensure reproducible staining with a final array of 36 cores.

Five micron thick microarray sections were prepared onto charged glass slides and deparaffinized in three washes of xylene and then through graded alcohol (100 to 90 to $70 \%$ ) to deionized water. Antigen retrieval consisted of incubating the tissue array sections in boiling $0.01 \mathrm{M}$ citrate buffer, $\mathrm{pH} 6.0$ for the indicated time and allowing the sections to cool to room temperature before staining. Staining was performed by a computer-controlled automated immunostainer (NexES, Ventana Medical Systems, Tucson, AZ) for which the staining protocols were optimized and programmed into the computer.

The antibodies, their dilutions and antigen retrieval (AR) times (in $\min$ ) used in this study were as follows: rabbit antihuman PDCD4 polyclonal antibody [1:100 dilution, AR (10)] (16), antihuman ER mouse monoclonal antibody - clone 6F11 [prediluted, AR (20), Ventana Medical Systems], antihuman PR mouse monoclonal antibody - clone 1A6 [prediluted, AR (20), Ventana Medical Systems], antihuman c-ErbB2 mouse monoclonal antibody - clone CB11 [prediluted, AR (10), Ventana Medical Systems] and rabbit antihuman RAR $\alpha$ polyclonal antibody [1:40 dilution, AR (20), Santa Cruz, USA]. The chromogen used in these stains is 3,3diaminobenzidine (DAB) resulting in a brown precipitate. Negative controls consisted of incubating the tissue section with isotype-matched serum without a primary antibody. The stained sections were then scored by HW and HY.

Evaluation of $\mathrm{PDCD}^{+}, \mathrm{ER}^{+}, \mathrm{PR}^{+}, \mathrm{HER} 2 / \mathrm{neu}^{+}$and $\mathrm{RAR} \mathrm{R}^{+}$cells. Assessment of immunohistochemical staining was performed jointly by two investigators (Y.H.W. and H.Y) using a double objective microscope. Homogeneous cytoplasmic or nuclear staining for PDCD4 was accepted as positive. The percentage of positive cells for PDCD4 was calculated by counting 2,000 tumor cells in the most positive areas and at least 10 highpower fields (HPFs; $0.16 \mathrm{~mm}^{2}$ ) in each case. Positive staining was graded as a percent of positive staining: $0=0-10 \%$, $1+=10-25 \%, 2+=26-50 \%$ and $3+=>50 \%$. Similar grading criteria were used to grade staining for ER, PR and RAR $\alpha$. The staining intensity for PDCD4 was assessed as being either weak or strong when compared to the staining intensity observed in normal breast epithelial cells. The mean intensity of staining was calculated as the mean intensity of staining observed in duplicate 3-mm cores taken from different areas of each case.

The established Dako scoring for erb-B2 was used: $0=<10 \%$ and weak with less than full membrane staining, $1=>10 \%$ but weak and less than full membrane staining, $2=>10 \%$ and weak with full membrane staining, $3=>10 \%$ and strong with full membrane staining.

Drugs. 5-deoxy-azacytidine (5-cd) was obtained from Sigma (St. Louis, MO, USA). T-47D, MDA-MB-231 and MDA- 
MB-435 cells were incubated with $0.1 \mu \mathrm{M}$ or $1 \mu \mathrm{M}$ 5-cd for $72 \mathrm{~h}$.

Western blotting. Cells were plated in $60-\mathrm{mm}$ tissue culture dishes at a density of $0.5 \times 10^{6}$ cells/dish in $5 \mathrm{ml}$ of $10 \%$ FCS supplemented Leibowitz's L-15 medium (for MDA-231 and MDA-MB-435 cells) or RPMI-1640 (for T-47D cells) containing either DMSO (vehicle used to dissolve 5-cd), or 5-cd at concentrations previously mentioned. Following incubation for $72 \mathrm{~h}$, the medium was removed and the cells were washed twice with PBS. Whole cell extracts were prepared by suspending the cells in lysis buffer containing $60 \mathrm{mM}$ Tris (pH 7.5), 7.5\% glycerol, 5\% 2-mercaptoethanol and 1\% SDS and the protein concentration was determined using the BCA assay (Bio-Rad Laboratories, Hercules, CA, USA). Extracts containing $25 \mu \mathrm{g}$ of protein were electrophoresed in $10 \%$ polyacrylamide-SDS denaturing gels and transferred to nitrocellulose membrane (Osmonics, Westborough, MA, USA) over $3 \mathrm{~h}$ by electroblotting. Ponceau S staining of the membrane and Coomassie blue staining of the SDS-PAGE gel were used to confirm that equal amounts of protein were transferred from each lane. The membrane was blocked with $5 \%$ non-fat milk in TBS [50 mM Tris ( $\mathrm{pH} 7.5)$ and $150 \mathrm{mM}$ $\mathrm{NaCl}$ ] for $1 \mathrm{~h}$ at room temperature. The membrane was then incubated with a rabbit polyclonal PDCD4 antibody at a 1:1000 dilution overnight at $4^{\circ} \mathrm{C}$. After washing with TBS, the membrane was incubated with goat anti-rabbit IgG conjugated with horseradish peroxidase (1:2000 dilution) for $1.5 \mathrm{~h}$ at room temperature. The membrane was then washed with TBS twice, followed by two washes with TBS containing $0.05 \%$ Tween-20 and developed using enhanced chemiluminescence (ECL) (Pierce, Rockford, IL, USA).

Statistical analysis. Statistical analysis was performed using the Prism Graphpad software (Graphpad Prism v4.0b, San Diego, CA, USA). ANOVA was used to compare multiple groups and the Bonferroni's multiple comparison tests were also calculated. $\mathrm{P}<0.05$ was considered statistically significant.

\section{Results}

Expression of PDCD4 in normal and malignant breast cells. PDCD4 was abundantly expressed as a cytoplasmic protein in all 5 cases of normal breast tissue (Fig. 2C, left panel). Benign epithelial cells adjacent to the tumor also showed an abundant PDCD4 expression in all 28 cases analyzed (Fig. 2C, middle and right panels). In contrast, PDCD4 was expressed at varying levels in $13(87 \%)$ of 15 DCIS and in only $36(55 \%)$ of 65 invasive ductal carcinoma cases (Fig. 1A). Some differences in the distribution and intensity of staining were observed between DCIS, DCIS with invasive ductal carcinoma and pure invasive ductal carcinoma. Specimens were assigned scores 0-3 according to the intensity of the staining and the number of stained cells. The difference of the PDCD4 expression in normal breast tissue compared with in situ and the invasive breast carcinomas is shown in Fig. 1B. When compared to normal breast tissue and DCIS, the invasive carcinoma showed a significantly reduced expression of PDCD4 (Fig. 1B). This is statistically significant with $\mathrm{p}<0.001$.
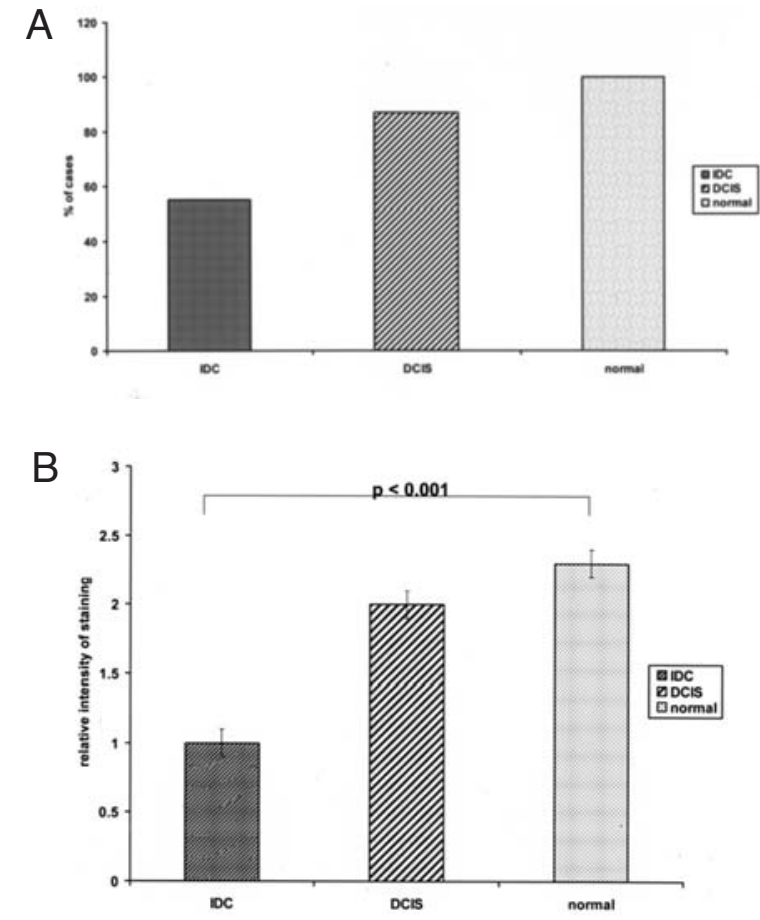

Figure 1. Graphical representation of the expression of PDCD4 (A) and relative intensity of PDCD4 staining (B) in archived breast samples (normal and malignant). The number of positive cases is shown as a percent of the total number of cases analyzed. (IDC, invasive ductal carcinoma; DCIS, ductal carcinoma in situ). The mean intensity of staining was calculated as the mean intensity of staining observed in duplicate $3-\mathrm{mm}$ cores taken from different areas of each case.

Subcellular localization of PDCD4 staining in breast cells. PDCD4 was present in both the cytoplasmic and nuclear subcellular compartments. In the benign breast epithelium, PDCD4 was predominantly localized within the cytoplasm (25 out of $28,89 \%$ ) (Fig. 2A-C). Nuclear staining for PDCD4 was noted in $10(36 \%)$ out of the 28 cases. Nuclear staining was not as intense as cytoplasmic staining in these cases. Weak cytoplasmic (1+) but stronger nuclear staining (2-3+) for PDCD4 was observed in 3 out of the 28 cases of the benign breast epithelium adjacent to the tumor that was analyzed. A greater proportion of DCIS showed nuclear staining for PDCD4. A nuclear PDCD4 expression was evident in 9 (60\%) out of the 15 DCIS. This observation was unique in DCIS (Figs. 2B and D). In invasive ductal carcinoma, both nuclear and cytoplasmic immunostaining for PDCD4 were reduced (Fig. 2D). Unlike DCIS, the localization of PDCD4 in the invasive carcinoma was observed more often in the cytoplasm (34 out of $65,52 \%$ ) rather than in the nucleus (14 out of 65 , 22\%) (Figs. 2B and D).

Correlation between PDCD4, ER, PR, HER2/neu and $R A R$ expression. In our previous study, looking at PDCD4 expression in cultured cells, we observed PDCD4 expression in all ER positive breast cancer cell lines analyzed. Therefore, we wanted to see if this relationship holds true in human breast cancer tissue samples. We analyzed the relationships between PDCD4 expression, estrogen receptor (ER) and HER2/neu status. Overall, 15 (19\%) cases were 
A

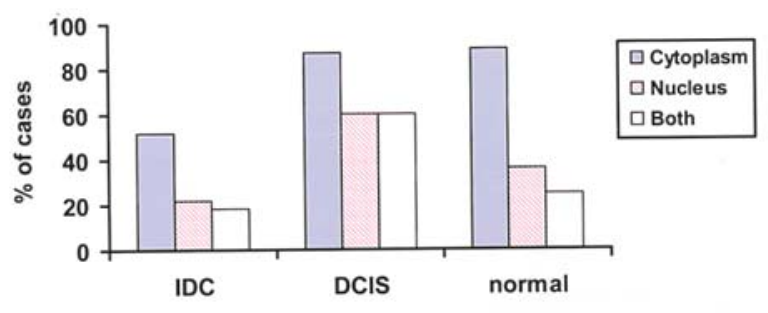

$\mathrm{B}$

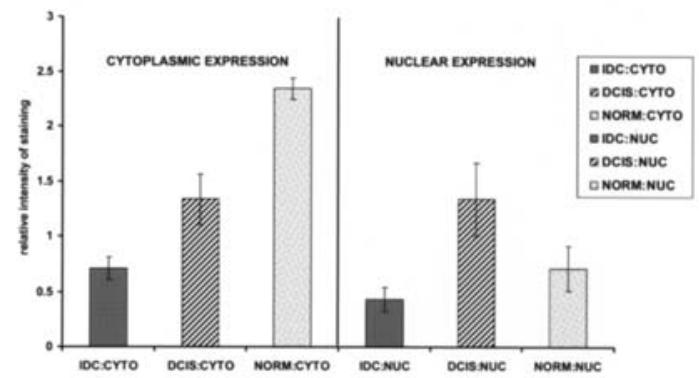

C

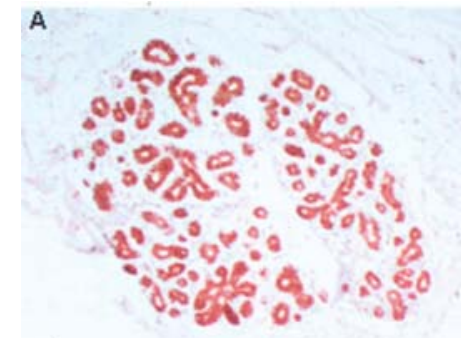

$\times 4$

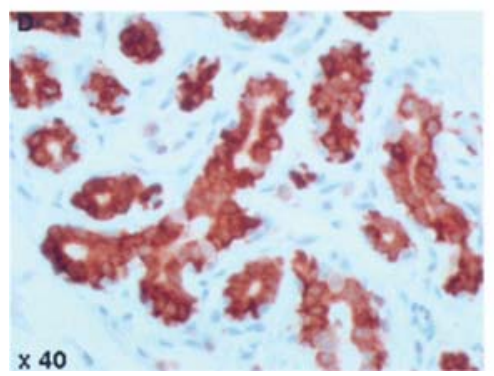

D

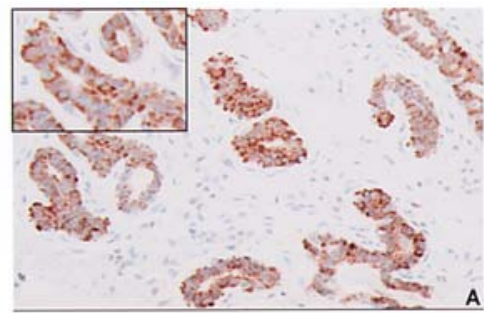

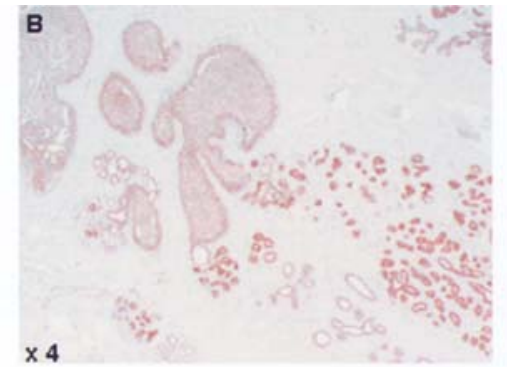
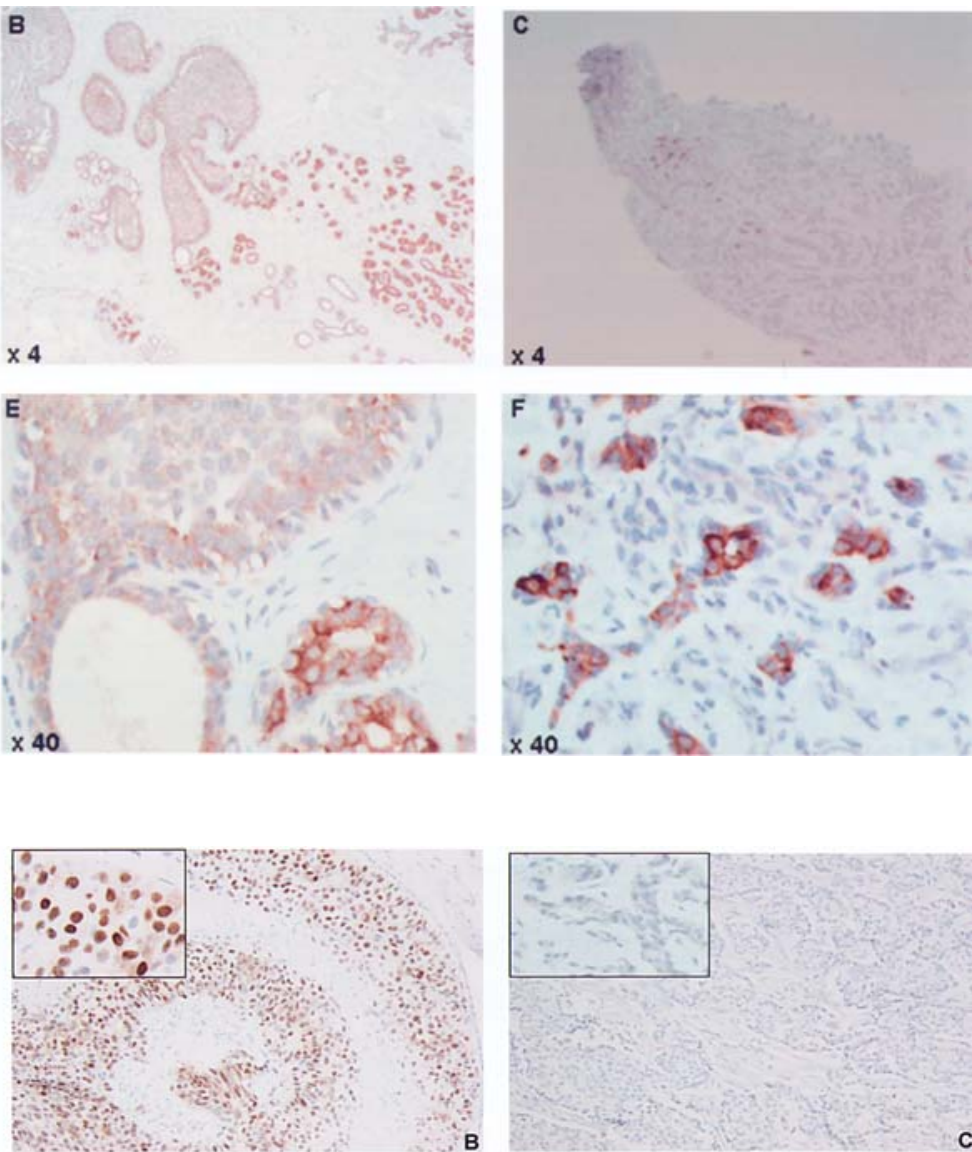

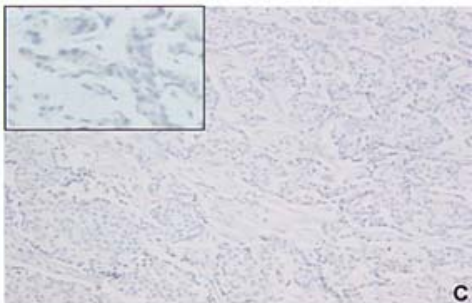

Figure 2. PDCD4 is expressed as a cytoplasmic and/or nuclear protein in normal and transformed breast cells. (A) a bar chart showing the number of cases (expressed as a percentage of the total number of cases analyzed) with cytoplasmic and/or nuclear expression of PDCD4. (B) a representation of the relative intensity of the cytoplasmic or nuclear expression of PDCD4 in normal breast epithelial cells (norm), ductal carcinoma in situ (DCIS) and invasive ductal cancer (IDC). (C) and (D) representative pictures of the immunohistochemical staining pattern for PDCD4 in normal (C) and transformed (D) breast cells. (C) [low magnification (A-C, upper panel) and high magnification (D-F, lower panel)] shows normal breast epithelial cells (left panel), normal breast epithelium adjacent to DCIS (middle panel) and normal breast epithelium adjacent to IDC (right panel). (D) representative cases of normal breast epithelial (A, left), DCIS with predominant nuclear staining (B, middle) and low staining of PDCD4 observed in IDC (C, right). The insert shows each section at a high magnification.

ER-/HER2/neu ${ }^{+}, 22(27 \%)$ cases were ER ${ }^{+} / \mathrm{HER} 2 / \mathrm{neu}^{+}, 21$ (26\%) cases were ER-/HER2/neu and 22 (27\%) cases were $\mathrm{ER}^{+} / \mathrm{HER} 2 / \mathrm{neu}^{-}$. There was a significant association between the expression of ER, HER2/neu and PDCD4 nuclear localization. PDCD4 was expressed as a nuclear protein in all DCIS cases that were ER+HER2/neu (Fig. 3A), whereas only $50 \%$ of ER-/HER2/neu DCIS tumors expressed a PDCD4 protein (Fig. 3A). An increased nuclear expression observed in $\mathrm{ER}^{+} / \mathrm{HER} 2 / \mathrm{neu}^{-}$DCIS cases was statistically significant when compared with $\mathrm{ER}^{+} / \mathrm{HER} 2 /$ neu $^{-}$IDC cases with $\mathrm{p}<0.05$
(Fig. 3A). When compared to ER ${ }^{+} / \mathrm{HER} 2-$ tumors (DCIS and IDC), a decreased expression of PDCD4 was observed in most ER-/HER2- tumors and this was statistically significant with $\mathrm{p}<0.03$ (Fig. 3C). We did not identify any cases with exclusive nuclear staining for PDCD4.

We did not observe any significant relationship between PDCD4 expression, RAR and PR status of breast cancer cells (data not shown). There were 5 cases of DCIS with IDC that were strongly positive for ER and PR (3+) and negative for HER2/neu. PDCD4 was expressed as a nuclear and/or 
A

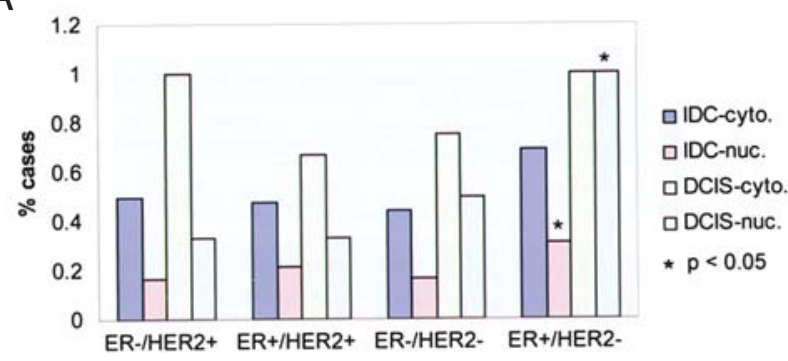

B

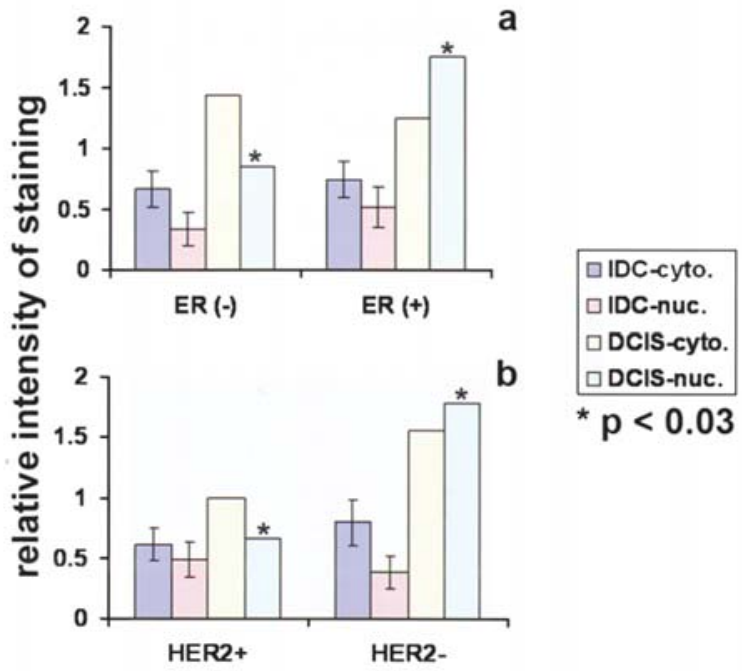

Figure 3. Correlation of PDCD4 expression with ER and HER2/neu expression. (A) shows a number of cases (represented as a percentage of the total cases analyzed) expressing PDCD4 either as a cytoplasmic and/or nuclear protein in relation to the expression of ER or HER2/neu. (B) the mean intensity of staining for PDCD4 in DCIS and IDC in relation to the ER (a) or Her2/neu (b) status.

cytoplasmic protein in areas of DCIS in all of these tumors, as opposed to the absence or very weak expression of PDCD4 in the IDC component of these tumors. The nuclear staining observed in the DCIS components was similar or greater to that observed within the cytoplasm of these cells. A weak cytoplasmic expression of PDCD4 was observed in 2 of the 3 $\mathrm{ER}^{+} / \mathrm{PR}-\mathrm{HER} 2-\mathrm{IDC}$ cases analyzed.

Effect of demethylating agent on PDCD4 expression in T-47D and MDA-MB-435 cells. DNA demethylation plays a critical role in transcriptional regulation in differentiated somatic cells and a loss or decreased gene expression in tumor cells can result from hypermethylation of $\mathrm{CpG}$ dinucleotides within the promoter region of the gene. The PDCD4 promoter is yet to be identified. An analysis of the 5' sequences immediately upstream of the start of transcription revealed several potential $\mathrm{CpG}$ methylation sites. Therefore, we sought to see if 5-aza-2'-deoxycytidine (5-cd) would induce PDCD4 expression in breast cancer cells. T-47D, MDA-MB-231 and MDA-MB-435 cells were treated with $100 \mathrm{~nm}$ and $1 \mu \mathrm{M} 5$-cd for $48 \mathrm{~h}$. Total cell lysate was isolated from these cells and analyzed by immunoblotting using a specific PDCD4 polyclonal antibody that was previously reported (2). PDCD4 protein expression was not induced by 5-cd in T-47D

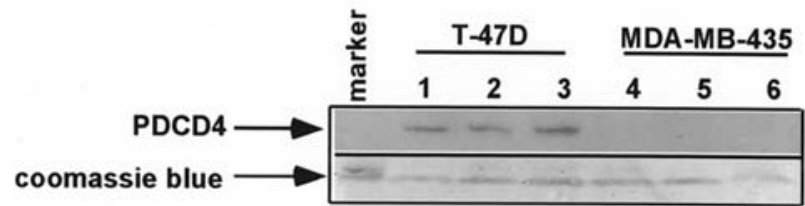

Figure 4. A lack of induction of the PDCD4 expression in breast cancer cells treated with 5-aza-deoxycytidine. T-47D (lanes 1-3), MDA-MB-435 (lanes 4-6) and MDA-MB-231 (not shown) were incubated with $100 \mathrm{nM}$ (lanes 2 and 5) or $1 \mu \mathrm{M}$ (lanes 3 and 6) 5-aza-cytidine for $72 \mathrm{~h}$. Immunoblotting for PDCD4 protein expression was performed on whole cell extract. Induction of PDCD4 expression was not detected in T-47D (lanes 2 and 3), MDA-MB-435 (lanes 5 and 6) and MDA-MB- 231 (not shown) breast cancer cells. Coomassie blue staining of the SDS-PAGE gel confirmed equal loading of protein.

and MDA-MB-435-cells as seen in Fig. 4. Similar results were obtained in the MDA-MB-231 cells (data not shown).

\section{Discussion}

The focus of this study was to analyze PDCD4 expression in breast tissues and determine the clinical significance of variations in PDCD4 expression in ductal carcinoma of the breast. Consideration of the in vivo pattern of the expression of PDCD4 in breast tissues and a better understanding of the mechanism of the action of PDCD4 will help in understanding its role in breast physiology and carcinogenesis.

The present study demonstrated that PDCD4 is abundantly expressed as a cytoplasmic protein in normal breast epithelial cells. In addition to the predominant cytoplasmic expression observed in normal breast epithelial cells, a weak nuclear expression was observed in $10 \%$ of cases analyzed. These findings are in agreement with those previously reported by Yoshinaga et al (2). The expression of PDCD4 was observed within the epithelial cells of normal breast tissue and it was observed in both the nucleus and the cytoplasm (2). These findings suggest that PDCD4 may play a role in normal breast physiology. We observed a marked decrease in the PDCD4 expression in transformed breast cells. In ductal carcinoma in situ, the expression of PDCD4 was observed in $87 \%$ of tumor samples analyzed, whereas only $55 \%$ of the invasive ductal carcinoma samples weakly expressed PDCD4. More importantly, the PDCD4 expression was significantly reduced in the invasive breast carcinoma when compared to normal breast epithelium and this was statistically significant $(p<0.001)$. Our findings suggest that a loss of the PDCD4 expression may contribute to breast carcinogenesis and may be associated with a more aggressive phenotype. These findings are similar to those recently reported by Zhang et al (16). In their series of 18 hepatocellular carcinoma samples, Zhang et al, observed reduced levels of the PDCD4 protein in hepatocellular carcinoma tissues when compared with their corresponding non-cancerous tissues (16). Chen et al also reported a decreased expression of PDCD4 in human lung cancers and this correlates with tumor progression and poor prognosis (17). Loss of the PDCD4 expression has also been implicated in the pathogenesis of glioblastoma multiforme (18). 
It was previously reported that PDCD4 shuttles between the nucleus and cytoplasm and may have a role in RNA metabolism in addition to its role in protein translation (7). In our study, we observed that PDCD4 is expressed primarily as a cytoplasmic protein in normal breast epithelial cells, with an increased nuclear expression seen in most cases of DCIS analyzed and a significantly decreased nuclear and cytoplasmic expression in most cases of IDC analyzed. In cultured breast cancer cells, PDCD4 is expressed primarily as a nuclear protein and an enforced expression of PDCD4 in these cells results in apoptosis. These observations suggest that changes in the cellular sub-localization of PDCD4 may be one of the contributing events in the process of breast carcinogenesis. It is tempting to speculate that the PDCD4's main physiological role in breast cancer cells involves the regulation of protein synthesis and signals resulting in uncontrolled cell proliferation that may result in an increased nuclear import to enhance its role in RNA metabolism, thereby resulting in a decreased synthesis of proteins that support uncontrolled growth and survival. It will be important to identify key proteins that regulate PDCD4 expression and its subcellular localization and how aberrations in these processes contribute to carcinogenesis.

We observed a significant correlation between the PDCD4 expression and ER. This is particularly of interest as it was previously shown that the antisense PDCD4-transfected MCF 7 breast cancer cells were less sensitive to the anti-proliferative effects of tamoxifen and geldanamycin (19). Additionally, PDCD4 was recently identified as an ER status reporter gene in a 'gene expression signature' that was associated with a better outcome (20). We speculate that PDCD4 may be involved in cellular responses mediated via ER. Although we had previously identified PDCD4 as a retinoid induced gene, we did not demonstrate any significant association between the PDCD4 and RAR expression in our series. One potential explanation for the findings observed in the current study is that we analyzed the basal expression of PDCD4 and not the induction of PDCD4 by RAR agonists. It should be noted that some RAR+ breast cancer cell lines had low basal or absent levels of the expression of PDCD4. However, when incubated with RAR agonists, PDCD4 expression was induced (15).

Unlike other classic tumor suppressor genes such as p53 and retinoblastoma genes, there are no published studies to show that PDCD4 is inactivated by methylation, deacetylation or genetic mutations in human cancers. Our study is the first to suggest that hypermethylation of $\mathrm{CpG}$ dinucleotides within the promoter region of the PDCD4 gene is not responsible for the lack of PDCD4 expression in breast cancer cells and this may apply to other cancers in which decreased expression of PDCD4 was documented. In cultured breast cancer cells treated with the histone deacetylase inhibitor, Trichostatin A, we did not observe any changes in the PDCD4 expression levels (data not shown), suggesting that chromatin remodeling does not directly influence PDCD4 expression in breast cancer cells. Other mechanisms of down-regulation of classic tumor suppressor genes include point mutations, a loss of heterozygosity and microdeletion of the region of DNA in which the gene is contained. PDCD4 is located on chromosome 10q24 and there is no published report of chromosomal aberrations involving this locus. There are published studies documenting allelic damage involving 10q in columnar cell changes with atypia, ductal carcinoma in situ and invasive carcinoma (21). The gene most frequently involved is the PTEN gene located on 10q23. Further studies specifically looking at the PDCD4 locus are required to determine if this region is also damaged during the progression from atypia to invasive carcinoma.

Our results indicate that PDCD4 is mainly expressed in the cytoplasm of normal breast cells. There is increased nuclear expression of PDCD4 in DCIS and a marked decrease in cytoplasmic and nuclear expression in IDC. PDCD4 is expressed in most of the ER positive tumors analyzed. Our data correlating cellular localization of PDCD4 with ER/HER2 status stem from the small number of DCIS cases we were able to obtain from the archival Surgical Pathology files. We had limited information as to the metastatic status of the archived tumors analyzed in this study. It will be of interest to know if a loss of the PDCD4 expression correlates with metastatic disease. Our findings support a role for PDCD4 in normal breast physiology and a role for the loss of the PDCD4 expression in breast carcinogenesis. Our findings also indicate that PDCD4 shuttles between the nuclear and cytoplasmic compartments of the cells and its subcellular localization will determine its role in cellular physiology. In addition, in cultured breast cancer cells, absence of the induction of PDCD4 expression by 5-cd suggests that hypermethylation of the PDCD4 promoter does not contribute to a decreased expression observed in breast cancer cells. Our future studies are aimed at identifying proteins that regulate PDCD4 and define the role of PDCD4 in the growth and biological behavior of normal and transformed breast cells.

\section{Acknowledgements}

We are grateful to Dr Angel Pellicer (Department of Pathology, NYU School of Medicine) for the critical review of this manuscript. This study was supported by the Robert Wood Johnson Foundation Grant (038398) (OA), NIH Grant K12CA01713 (OA) and an Irma T. Hirschl Charitable Trust Award (C015710) (OA).

\section{References}

1. Matsuhashi S, Yoshinaga H, Yatsuki H, Tsugita A and Hori K: Isolation of a novel gene from human cell line with Pr-28 MAb which recognizes a nuclear antigen involved in the cell cycle. Res Commun Biochem Cell Mol Biol 1: 109-120, 1997.

2. Yoshinaga H, Matsuhashi S, Fujiyama C and Masaki Z: Novel human PDCD4 (H731) gene expressed in proliferative cells is expressed in the small duct epithelial cells of the breast as revealed by an anti-H731 antibody. Pathol Int 49: 1067-1077, 1999.

3. Cmarik JL, Min H, Hegamyer G, Zhan S, Kulesz-Martin M, Yoshinaga H, Matsuhashi $\mathrm{S}$ and Colburn NH: Differentially expressed protein Pdcd4 inhibits tumor promoter-induced neoplastic transformation. Proc Natl Acad Sci USA 96: 14037-14042, 1999.

4. Yoshinaga H, Matsuhashi S, Ahanek J, Masaki Z and Hori K: Expression and identification of H731 gene product in HeLa cells. Res Commun Biochem Cell Mol Biol 1: 121-131, 1997.

5. Yang HS, Jansen AP, Komar AA, Zheng X, Merrick WC, Costes S, Lockett SJ, Sonenberg N and Colburn NH: The transformation suppressor Pdcd4 is a novel eukaryotic translation initiation factor $4 \mathrm{~A}$ binding protein that inhibits translation. Mol Cell Biol 23: 26-37, 2003. 
6. Soejima H, Miyoshi O, Yoshinaga H, Masaki Z, Ozaki I, Kajiwara S, Niikawa N, Matsuhashi S and Mukai T: Assignment of the programmed cell death 4 gene (PDCD4) to human chromosome band 10q24 by in situ hybridization. Cytogenet Cell Genet 87: 113-114, 1999.

7. Bohm M, Sawicka K, Siebrasse JP, Brehmer-Fastnacht A, Peters $\mathrm{R}$ and Klempnauer KH: The transformation suppressor protein Pdcd4 shuttles between nucleus and cytoplasm and binds RNA. Oncogene 22: 4905-4910, 2003.

8. Shibahara K, Asano M, Ishida Y, Aoki T, Koike T and Honjo T: Isolation of a novel mouse gene MA-3 that is induced upon programmed cell death. Gene 166: 297-301, 1995.

9. Jurisicova A, Latham KE, Casper RF and Varmuza SL: Expression and regulation of genes associated with cell death during murine preimplantation embryo development. Mol Reprod Dev 51: 243-253, 1998.

10. Yang HS, Jansen AP, Nair R, Shibahara K, Verma AK, Cmarik JL and Colburn NH: A novel transformation suppressor, Pdcd4, inhibits AP-1 transactivation but not NF-kappaB or ODC transactivation. Oncogene 20: 669-676, 2001.

11. Palamarchuk A, Efanov A, Maximov V, Aqeilan RI, Croce CM and Pekarsky Y: Akt phosphorylates and regulates Pdcd4 tumor suppressor protein. Cancer Res 65: 11282-11286, 2005.

12. Yang HS, Matthews CP, Clair T, Wang Q, Baker AR, Li CC, Tan TH and Colburn NH: Tumorigenesis suppressor pded4 downregulates mitogen-activated protein kinase kinase kinase kinase 1 expression to suppress colon carcinoma cell invasion. Mol Cell Biol 26: 1297-1306, 2006.

13. Lankat-Buttgereit B, Gregel C, Knolle A, Hasilik A, Arnold R and Goke R: Pdcd4 inhibits growth of tumor cells by suppression of carbonic anhydrase type II. Mol Cell Endocrinol 214: 149-153, 2004.
14. Azzoni L, Zatsepina O, Abebe B, Bennett IM, Kanakaraj P and Perussia B: Differential transcriptional regulation of CD161 and a novel gene, 197/15a, by IL-2, IL-15 and IL-12 in NK and T cells. J Immunol 161: 3493-3500, 1998.

15. Afonja O, Juste D, Das S, Matsuhashi S and Samuels HH: Induction of PDCD4 tumor suppressor gene expression by RAR agonists, Anti-estrogen and HER2/neu antagonist in breast cancer cells. Evidence for a role in apoptosis. Oncogene 23: 8135-8145, 2004.

16. Zhang H, Ozaki I, Mizuta T, Hamajima H, Yasutake T, Eguchi Y, Ideguchi $\mathrm{H}$, Yamamoto $\mathrm{K}$ and Matsuhashi S: Involvement of programmed cell death 4 in transforming growth factor-beta1induced apoptosis in human hepatocellular carcinoma. Oncogene 00: 1-12, 2006.

17. Chen Y, Knosel T, Kristiansen G, Pietas A, Garber ME, Matsuhashi S, Ozaki I and Petersen I: Loss of PDCD4 expression in human lung cancer correlates with tumor progression and prognosis. J Pathol 200: 640-646, 2003.

18. Gao F, Zhang P, Zhou C, Li J, Wang Q, Zhu F, Ma C, Sun W and Zhang L: Frequent loss of PDCD4 expression in human glioma: possible role in the tumorigenesis of glioma. Oncol Rep 17: 123-128, 2007.

19. Jansen AP, Camalier CE, Stark C and Colburn NH: Characterization of programmed cell death 4 in multiple human cancers reveals a novel enhancer of drug sensitivity. Mol Cancer Ther 3: 103-110, 2004.

20. van't Veer LJ, Dai H, van de Vijver MJ, et al: Gene expression profiling predicts clinical outcome of breast cancer. Nature 415: 530-536, 2002.

21. Dabbs DJ, Carter G, Fudge M, Peng Y, Swalsky P and Finkelstein S: Molecular alterations in columnar cell lesions of the breast. Mod Pathol 19: 344-349, 2006. 